\title{
Brussels Sprout
}

National Cancer Institute

\section{Source}

National Cancer Institute. Brussels Sprout. NCI Thesaurus. Code C71998.

A leafy green vegetable and a member of the Gemmifera group of cabbages that contains high amounts of phytochemicals, with potential chemopreventive activity. Brussels sprouts contain high amounts of the glycosinolate glucobrassicin. Upon consumption of Brussels sprouts, the phytochemicals in the vegetable, and some of their metabolites, are able to induce the expression of various hepatic cytochrome P450 monooxygenases, scavenge free radicals and may inhibit the activation of various signal transduction pathways. 